(C) 1982. The Genetical Society of Great Britain

\title{
THE EFFECT OF GENETIC BACKGROUND ON THE FITNESS OF DIAZINON RESISTANCE GENOTYPES OF THE AUSTRALIAN SHEEP BLOWFLY, LUCILIA CUPRINA
}

\author{
J. A. MCKENZIE, M. J. WHITTEN* and M. A. ADENA $\dagger$ \\ Department of Genetics, University of Melbourne, Victoria, Parkville 3052; † Depart- \\ ment of Population Biology, R.S.B.S., A.N.U., Canberra, A.C.T. Australia
}

Received 28.xii.81

\section{SUMMARY}

\begin{abstract}
Laboratory and field experimentation has shown that resistant and susceptible diazinon genotypes of flies collected from the field may have similar fitness in an environment free of diazinon. If the genetic background of resistant genotypes from the field is disrupted, the fitness of the resistant genotype declines. These results, in conjunction with previous data, indicate a modification of the genetic background in field populations following the spread of the resistance allele some ten years earlier. It is suggested that this outcome is dependent on the availability of genetic variability, the intensity of selection and the duration of insecticide usage after resistance develops.
\end{abstract}

\section{INTRODUCTION}

THE evolution of insecticide resistance of economic importance may usually be described in terms of allelic changes at a single genetic locus (Brown and Pal, 1971; Georghiou and Taylor, 1977). However, prolonged selection by the insecticide after resistance has become widespread may result in an increased relative fitness in the absence of the insecticide (Bøggild and Keiding, 1958; Abedi and Brown, 1960; Keiding, 1967; White, 1978).

Resistance to diazinon, an organophosphorous insecticide widely used in Australia for blowfly control, was reported in Lucilia cuprina in 1967, approximately ten years after the introduction of the insecticide (Shanahan, 1967). Arnold and Whitten (1976) and McKenzie et al. (1980) have demonstrated that resistance could be largely explained by allelic changes at a locus on the fourth chromosome. In the field, L. cuprina is the primary cause of flystrike or myiasis in sheep (Foster et al., 1975). However, in the laboratory, L. cuprina is readily amenable to experimentation (Whitten et al., 1975). Arnold and Whitten (unpublished) conducted population cage studies in 1969/70, comparing the fitness of susceptible $(++)$, intermediate $(+R)$ and resistant $(R R)$ genotypes. They found that, in the absence of insecticide, the $R$ allele was eliminated at a rate consistent with its being an additive deleterious.

Field surveys in Victoria in $1977 / 78$ revealed L. cuprina populations to be close to fixation for the resistance allele irrespective of insecticide usage and sheep management practices in the sampled areas (McKenzie et al., 1980). While there is evidence that selection for resistance may occur at low residual concentrations of the insecticide on sheep (Shanahian and

\footnotetext{
* Present address: Division of Entomology, CSIRO, P.O. Box 1700, Canberra City 2601.
} 
Roxburgh, 1974; Hart et al., 1979; McKenzie and Whitten, 1982), the high $R$ allele frequencies in areas of restricted insecticide usage seemed at variance with Arnold and Whitten's population cage results. For this reason, experiments were commenced to test the relative fitness of diazinon resistant genotypes in the absence of insecticide. Our aim was to decide whether extensive use of diazinon over the previous decade had produced changes in fitness due to modification of the genetic background associated with the resistant genotype.

The opportunity to carry out any genetic analysis of background modifications of a resistance locus in a field population occurs infrequently because the widespread occurrence of resistance in a pest species usually results in the rapid replacement of the now less-effective compound by a chemical to which the insect lacks cross resistance.

\section{MATERIAls AND METHOdS}

\section{(i) Strains}

The strains used were a standard reference susceptible strain, SWT, having the genotype ++ with respect to the diazinon resistance locus $R_{1}$ (Foster et al., 1981) and a pure breeding resistant strain, $R R$, derived from flies collected in 1979 from Bairnsdale in south eastern Victoria (McKenzie et al., 1980). The resistance phenotype of individuals from this strain is consistent with its being fixed for $\boldsymbol{R}_{1 A}$ (Arnold and Whitten, 1976; McKenzie et al., 1980). In the field experiments, the resistant strain was derived from $R \boldsymbol{R}$ but was also homozygous for dieldrin resistance. Diazinon and dieldrin resistance are toxicologically (McKenzie and Whitten, 1982) and genetically (Foster et al., 1981) independent in L. cuprina.

\section{(ii) Laboratory experiments}

\section{(a) Establishment of population cages}

\section{Insertion of wild type genetic background into $\mathrm{RR}$ genotype}

The $R R$ and SWT strains were crossed and $\mathrm{F}_{1}$ individuals used to establish a population cage (generation 0 of backcrossing). Virgin $F_{1}$ females were also backcrossed to SWT males and the $R+$ female progeny class was determined by the application of $0.5 \mu$ l of 0.01 per cent $(\mathrm{v} / \mathrm{v})$ diazinon. This dose does not discriminate with complete reliability between the ++ and $R+$ genotypes (see table 1 ). The fact that ca. 7 per cent of crosses contributing to the following generation of backcrosses was of the type

\section{TABLE 1}

Mortality levels* for the diazinon resistance genotypes following topical treatment of adults with $0.5 \mu l$ diazinon

$\begin{array}{cccc}\begin{array}{c}\text { Insecticide } \\ \text { concentration }(\mathrm{v} / \mathrm{v})\end{array} & ++ & \text { Genotype } R+ & \boldsymbol{R} R \\ 0.01 \% & 185 / 200 & 5 / 200 & - \\ 0.025 \% & - & 220 / 240 & 49 / 500\end{array}$

\footnotetext{
* Representative pooled data from a series of separate trials.
} 
$++x++$, was not considered important so long as the frequency of the $R+$ genotype was near expectation (50 per cent) at the commencement of the various cage experiments. The percentage mortality of each generation in the backcross programme was consistent with this expectation so that the lack of a fully discriminatory dose to separate ++ and $R+$ genotypes was not considered vital. The surviving virgin females of each generation were then backcrossed to SWT males with the procedure being repeated for nine generations of backcrossing. After three, six and nine generations of backcrossing, population cages were established using $R+$ individuals. The initial frequency of the + allele was therefore ca. 0.5 in each case.

\section{Re-introduction of RR background into RR genotype}

$\mathrm{F}_{1}$ females from the above $R \boldsymbol{R} \times \mathrm{SWT}$ cross were backcrossed to $R \boldsymbol{R}$ males. Virgin female progeny of this cross were individually mated to $R R$ males. Individual eggs were isolated and the genotype of each female determined using $0.5 \mu \mathrm{l}$ of 0.025 per cent $(\mathrm{v} / \mathrm{v})$ diazinon. A female killed by exposure to this dose of diazinon was classified as $R+$. Eggs from designated $R+\times R R$ matings were used to establish the next backcross generation. Clearly there exists the possibility that an $R R$ female could die either due to this insecticide treatment or from some other cause and hence be misclassified as $R+(c a .10$ per cent, see table 1$)$. Her progeny would all be $R R$ but would have received the appropriate $R R$ background along with the "legitimate" $R+\times R R$ progeny which had been pooled together to provide adults for the following generation. The possible misclassification of an $R R \times R R$ cross as $R+\times R R$ cross constitutes no major difficulty for the overall experiment so long as $R+$ progeny are adequately represented in any one generation. Since the 0.025 per cent treatment killed approximately 50 per cent of individuals in each generation, the procedure adopted for incorporating the $R R$ background was considered to be satisfactory.

Following three, six and nine generations of backcrossing, population cages were established with progeny from $R+\times R R$ crosses. At generation 0 of backcrossing a population was established with equal proportions of $R+$ and $R R$ flies. The expected initial frequency of the + allele was 0.25 in each case.

\section{(b) Population cages}

Each population cage was started with 40 flies of each sex and flies were maintained under normal laboratory conditions (Whitten et al., 1975) in a discrete generation design. Samples of 40 males and females were randomly chosen at each generation to continue the population cage. $\mathrm{A}$ further random sample of 100 females per generation was tested with $0.5 \mu l$ of 0.01 per cent $(\mathrm{v} / \mathrm{v})$ diazinon to determine the proportion of ++ individuals in the population. Replicate cages were established for each backcross regime.

\section{(c) Effect of larval density on competitiveness of genotypes}

Cultures of $\mathrm{F}_{2}$ eggs from the cross $++\times R R$ were reared on liver at three density levels $(400,2000$ and 4000 eggs per $700 \mathrm{~g}$ of sheep liver). 
Each culture was supplemented with extra liver $(350 \mathrm{~g})$ after 48 and 72 hours of subsequent development. Samples of 100 adult males at each density were treated with discriminating doses of diazinon to determine genotypic frequencies. In the experiment, those individuals who died within $24 \mathrm{hrs}$ following $0.5 \mu \mathrm{l}$ of 0.01 per cent diazinon were classified as ++ . The survivors were treated with $0.5 \mu l$ of 0.025 per cent diazinon and the survivors deemed to be $R R$, those dying being classified as $R+$. Two trials were conducted at each density level.

\section{(iii) Field experiments}

Merino sheep that had not been treated with diazinon for at least 12 months were used in this experiment. Artificial implants, following the techniques of McKenzie and Whitten (1982), using $F_{2}$ eggs from a cross between ++ and the derived $R R$ strain, were made on to sheep (implant 1, November 1979, two sheep; implant 2, December 1979, six sheep) and the genotypes of individuals reaching the adult stage ascertained as described above.

\section{RESULTS}

\section{(i) Laboratory experiments}

\section{(a) Population cages}

There are obvious differences in the proportion of ++ individuals in population cages. These differences may be related to the genetic background of the original population (fig. 1). A parsimonious description of the first ten generations of the data is given in the figure. The analysis was performed using the program GLIM3 (Baker and Nelder, 1978), which performs iterative weighted least squares calculations on the logistic transformed proportions.

In the first generation, all cultures from the backcrossing to ++ had ++ frequencies consistent with the theoretical $F_{2}$ frequency of $0.25\left(\chi_{8}^{2}=\right.$ $2 \cdot 76, p>0 \cdot 5)$. However, at the second generation, the ++ frequency was 0.35 , significantly higher than $0.25\left(\chi_{1}^{2}=266, p<0.001\right)$, and this frequency was homogeneous for all backcross regimes $\left(\chi_{3}^{2}=7 \cdot 2, p>0 \cdot 05\right)$. For nought and three generations of backcrossing, the ++ frequency remained at this level, showing only sampling variability (linear $\chi_{2}^{2}=2 \cdot 4$, $p>0.05)$. However, for six and nine generations of backcrossing the ++ frequency increased with time (linear $\chi_{2}^{2}=727, p<0 \cdot 001$ ), with the latter showing the greater rate of increase. There was only weak evidence that the rate of increase was not constant (quadratic $\chi_{2}^{2}=8.9,0.01<p<0.05$ ). For each degree of backcrossing, the replicates were homogeneous $\left(\chi_{6}^{2}=\right.$ 9.3, $p>0 \cdot 05$ ).

For nought, three and six generations of backcrossing to $R R$, the ++ frequency was independent of time (linear $\chi_{3}^{2}=1 \cdot 1, p>0 \cdot 5$ ), but for the culture begun after nine generations of backcrossing the ++ frequency decreased with time (linear $\left.\chi_{1}^{2}=29 \cdot 3, p<0.001\right)$. The different degrees of backcrossing showed significantly different frequencies at the first generation $\left(\chi_{3}^{2}=49.0, p<0.001\right)$, decreasing from nought to three to six generations of backcrossing (with nine usually lower again). However, the mean 


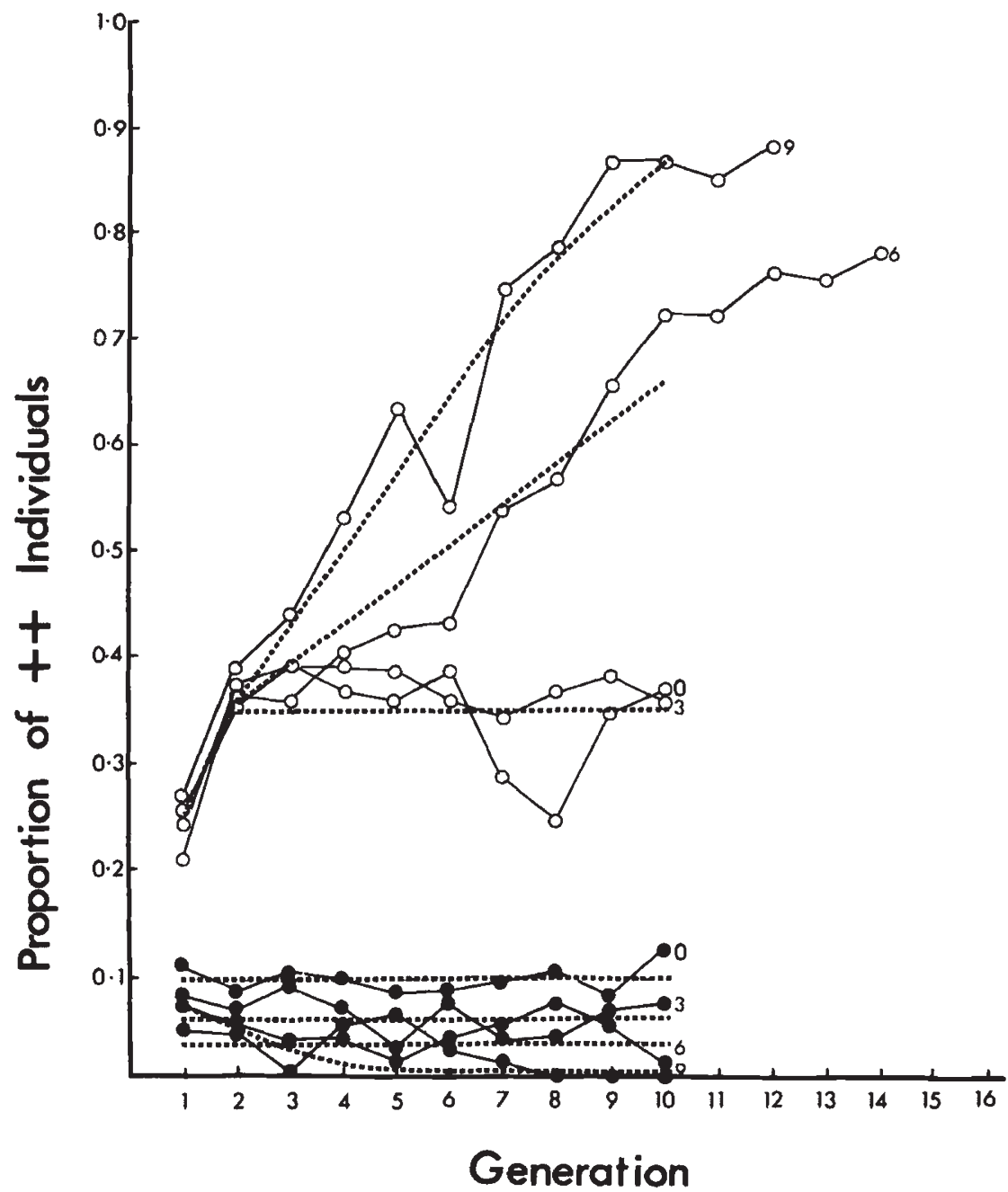

FIG. 1.-Proportion of ++ genotypes, averaged over 2 replicates, at each generation in population cages started following $0,3,6$ and 9 generations of backcrossing to $++(\mathrm{O}-\mathrm{O})$ or $R R(-1)$ strains. Lines of best fit (-- ) for the first 10 generations are given for each comparison, the 0,3 backcross to ++ comparisons having equivalent lines.

frequency was consistent with the theoretical $F_{2}$ frequency of 0.0625 $\left(\chi_{1}^{2}=0 \cdot 3, p>0 \cdot 5\right)$. The differences may have been caused by chance events in the setting up of the backcrosses. The replicates were homogeneous for each degree of backcrossing $\left(\chi_{5}^{2}=4 \cdot 8, p>0.05\right)$.

Extensive testing of the nine generations of backcrossing after ten generations in the population cages showed heterozygotes to be present. The frequency of the + allele was 0.06 .

(b) Effect of larval density

Larval density did not significantly influence the survival of genotypes (table 2). Only chance departure from the expected ratios was observed 
TABLE 2

Observed numbers (pooled over trials) of,$+++R$ and $\mathrm{R} R$ genotypes amongst $F_{2}$ adults derived from 3 egg densities on liver

$\begin{array}{rccccc} & ++ & +R & R R & \chi_{2}^{2} & P \\ 400 & 39 & 103 & 58 & 3.79 & 0 \cdot 2>P>0.1 \\ 2000 & 42 & 115 & 43 & 4.51 & 0 \cdot 2>P>0.1 \\ 4000 & 50 & 103 & 47 & 0.27 & 0.9>P>0.8\end{array}$

Contingency $\chi_{4}^{2}=4 \cdot 81,0 \cdot 40>P>0 \cdot 30$.

at each density and genotypes had similar developmental proportions across densities. The data are consistent with the assumption that genotypes have equal viability from the egg to the adult stage of the life cycle.

\section{(ii) Field experiments}

The above mentioned equality of viability in the absence of insecticide is supported by the field experiment. On sheep not treated with diazinon, egg to adult viability is independent of genotype (table 3 ). This has proved to be a general result in more extensive trials with artificial myiases (McKenzie and Whitten, 1982).

TABLE 3

Numbers of,$++ \mathbf{R}+$ and $\mathbf{R}$ genotypes amongst $F_{2}$ adults derived from eggs implanted on to sheep not treated with diazinon

$\begin{array}{lccccc} & ++ & +R & R R & \chi_{2}^{2} & P \\ \text { Implant 1 } & 30 & 43 & 19 & 3.02 & 0.3>P>0.2 \\ \text { Implant 2 } & 59 & 111 & 73 & 3.43 & 0.2>P>0.1 \\ \text { Total } & 89 & 154 & 92 & 2.23 & 0.4>P>0.3 \\ \text { Heterogeneity } & & & & 4 \cdot 22 & 0.2>P>0.1\end{array}$

\section{Discussion}

The time taken for resistance to develop subsequent to the introduction of diazinon (1958-1967), coupled with the fact that Whitten and Arnold (unpublished) found in 1969 that the $R R$ genotype was strongly selected against in the absence of diazinon in laboratory cage experiments, supports the view that $\boldsymbol{R}$-bearing individuals were initially at a selectively disadvantage in the field in the absence of the insecticide. The present results for both laboratory and field experimentation indicate similar egg to adult viability values for,$+++R$ and $R R$ genotypes (tables 2 and 3 ). It is at this stage of the life cycle that selection, with respect to resistance is most likely to act (Whitten et al., 1980). However, in population cage studies, which are dependent on all stages of the life cycle, populations derived directly from a cross between a susceptible laboratory strain $(++)$ and a resistant field strain $(R R)$ also showed the genotypes to have similar fitness (fig. 1). Furthermore, if the $R$ allele is placed progressively into a + background the population cage results are more in accord with those of 
Whitten and Arnold (loc. cit.). This backcrossing procedure would disrupt any co-adapted field genome.

In its simplest form, this co-adapted genome may involve a single linked modifier but it could also involve a complex of modifiers distributed throughout the genome. One way of distinguishing between these hypotheses is to carry out a reciprocal backcrossing regime (e.g., ++ female $\times R+$ male) to that already used to negate the possibility of recombination between any linked modifier and the resistance locus, as crossing over does not normally occur in males of $L$. cuprina (Foster et al., 1981). In population cages established following such a backcrossing regime similar results to those of the present experiment would be expected for the "whole genome" model. The fitness of the genotypes should be unaffected by backcrossing in the case of the linked modifier model so population cage results should be similar irrespective of the degree of backcrossing, if this model applies.

Regardless of the actual genetic mechanism it is clear that the backcrossing regime used in the current experiments enables a reconstruction of a genetic background that is presumably similar to that in which Whitten and Arnold's experiments were carried out. Progressive reconstruction of the field background by backcrossing to $R R$ does not have as marked an effect. There is an indication that ++ genotypes may not be as fit as the other two genotypes in the population cage experiment. Whether this particular result can be directly related to the field is not the point at issue. Taken in conjunction with the other data it seems reasonable to conclude that individuals carrying $R$ alleles are not at the disadvantage in a nondiazinon environment that they were a decade ago.

A resistance allele often produces marked physiological and biochemical disruption when it first arises, placing carriers at a selective disadvantage in environments free of insecticide (Brown and Pal, 1971). The initial deleterious effects on the occurrence of a resistance allele are not necessarily reflected in all stages of the life cycle (Pimental et al., 1951; Underhill and Merrell, 1966). Subsequent selection will favour modifications that minimize the deleterious effect of a new mutation, as is attested by the slow reversion rates of resistance in the absence of insecticides in some insect strains (Abedi and Brown, 1960; Forgash and Hansens, 1967).

In the present study it is likely that modification of the genetic background in the field genome has decreased the deleterious effects associated with the $R$ allele, resulting in the observed change in relative fitness. Rapid modification of the fitness of different strains involved in competitive interaction has been reported previously (Seaton and Antonovics, 1967; Ayala, 1969; Bryant and Turner, 1972; White, 1978; Hartl and Jungen, 1979). Inter- and intra-specific larval competition is likely in natural populations of $L$. cuprina (Foster et al., 1975).

Clearly, there will be instances where such modifications of the background do not occur, presumably because genetic variability is unavailable or the intensity or the duration of selection has been restricted. For example, in the case of dieldrin resistance in $L$. cuprina the latter is most likely because of the limited period of usage of that insecticide (Shanahan and Hughes, 1979). However, if models of the evolution of insecticide resistance are to be realistic, information of temporal changes in the relative fitness of genotypes is essential (McKenzie and Whitten, 1982). Keiding (1967) 
has argued that the longer there is selection for resistance, the greater will be the fitness of resistance genotypes. The present data support this contention; the consequence being of particular relevance to both fundamental and applied aspects of the evolution of insecticide resistance and, more generally, to questions of gene frequency and genome structure changes under different selection regimes.

Acknowledgments.-The authors would like to acknowledge Ms N. Austin for technical assistance, and Mr J. T. Arnold, Dr J. Dearn and Dr J. Oakeshott for useful comments on the text. Financial assistance for the project came from the Australian Research Grants Committee and the Australian Wool Corporation.

\section{REFERENCES}

ABEDI, Z. H., AND BROWN, A. W. A. 1960. Development and reversion of DDT-resistance in Aedes aegypti. Can. J. Genet. Cytol., 2, 252-261.

ARNOLD, J. T. A., AND WHITTEN, M. 3. 1976. The genetic basis for organophosphorus resistance in the Australian sheep blowfly, Lucilia cuprina (Wiedemann) (Diptera, Calliphoridae). Bull. Entomol. Res., 66, 561-568.

AYALA, F. J. 1969. Evolution of fitness. IV. Genetic evolution of inter-specific competitive ability in Drosophila. Genetics, 61, 737-747.

BAKER, R. J., AND NELDER, J. A. 1978. The GLDN system. Release 3. Generalised linear interactive modelling. NAG: London.

BøGGILD, I., AND KEIDING, J. 1958. Competition in house fly larvae. Experiments involving a DDT-resistant and a susceptible strain. Oikos, 9, 1-25.

BROWN, A. W. A., AND PAL, R. 1971. Insecticide resistance in arthropods. W.H.O. Geneva.

BRYANT, E. H., AND TURNER, C. R. 1972. Rapid evolution of competitive ability in larval mixtures of the house fly. Evolution, 26, 161-170.

FORGASH, A. J., AND HANSENS, E. F. 1967. Resistance levels in diazinon-pressured and non-pressured polyresistant house flies. J. Econ. Entomol., 60, 1241-1247.

FOSTER, G. G., KITCHING, R. L., VOGT, W. G., AND WHITTEN, M. J. 1975. Sheep blowfly and its control in the pastoral ecosystem of Australia. Proc. Ecol. Soc. Aust., 9, 213-229.

FOSTER, G. G., WHITTEN, M. J., KONOVAlOV, C., ARNOLD, J. T. A. AND MAFFI, G. 1981. Autosomal genetic maps of the Australian sheep blowfly, Lucilia cuprina dorsalis R.-D. (Diptera: Calliphoridae), and possible correlations with the linkage maps of Musca domestica L. and Drosophila melanogaster (Mg.). Genet. Res., 37, 55-69:

GEORGHIOU, G. P., AND TAYLOR, C. E. 1977. Genetic and biological influences in the evolution of insecticide resistance. J. Econ. Entomol., 70, 319-323.

HART, R. J., CAVEY, W. A., AND RYAN, K. J. 1979. Efficiency of insecticides for flystrike control especially under severe fly wave conditions. In National Symposium on Sheep Blowfly and Flystrike in Sheep. Department of Agriculture, N.S.W., pp. 79-95.

HARTL, D. L., AND JUNGEN, H. 1979. Estimation of average fitness of populations of Drosophila melanogaster and the evolution of fitness in experimental populations. Evolution, 33, 371-380.

KEIDING, J. 1967. Persistence of resistant populations after relaxation of the selection pressure. World Rev. Pest Control, 6, 115-130.

McKENZIE, J. A., DEARN, J. M., AND WHITTEN, M. J. 1980. Genetic basis of resistance to diazinon in Victorian populations of the Australian sheep blowfly, Lucilia cuprina. Aust. J. Biol. Sci., 33, 85-95.

McKeNZIE, J. A., AND WHITTEN, M. J. 1982. Selection for insecticide resistance in the Australian sheep blowfly, Lucilia cuprina. Experientia, 38, 84-85.

PIMENTAL, D., DEWEY, J. E., AND SCHWARDT, H. H. 1951. An increase in the duration of the life cycle of DDT-resistant strains of the house fly. J. Econ. Entomol., 44, 477-481.

SEATON, A. P. C., AND ANTONOVICS, J. 1967. Population interrelationships. I. Evolution in mixtures of Drosophila mutants. Heredity, 22, 19-34.

Shanahan, G. J. 1967. The sheep blowfly's tolerance to insecticides. Agric. Gaz. N.S.W., $78,444-445$.

SHANAHAN, G. J., AND ROXBURGH, N. A. 1974. Reduction in the period of protection from artificial flystrike by organophosphorus and organophosphorus-carbamate resistant larvae of Lucilia cuprina. Aust. Vet. J., 50, 177-178. 
SHANAHAN, G. J., AND HUGHES, P. B. 1979. The history and significance of insecticide resistance in field populations of the primary sheep blowfly, Lucilia cuprina. In National Symposium on Sheep Blowfly and Flystrike in Sheep. Department of Agriculture, N.S.W., pp. 69-78.

UNDERHILL, J. C., AND MERRELL, D. J. 1966. Fecundity, fertility, and longevity of DDTresistant and susceptible populations of Drosophila melanogaster. Ecology, 47, 140-142.

WHITE, R. M. 1978. Ecological and population genetics of insecticide resistance in the Australian sheep blowfly, Lucilia cuprina. Thesis, University of Liverpool.

WHITTEN, M. J., DEARN, J. M., AND McKENZIE, J. A. 1980. Field studies on insecticide resistance in the Australian sheep blowfly, Lucilia cuprina. Aust. J. Biol. Sci., 33, 725-735.

WHITTEN, M. J., FOSTER, G. G., ARNOLD, J. T. A., AND KONOVALOV, C. 1975. The Australian sheep blowfly, Lucilia cuprina. In R. C. King (ed.) Handbook of Genetics, vol. 3, Plenum, New York, pp. 401-418. 